\title{
Shifting home energy consumption through a holistic understanding of the
}

\section{home system of practice}

Christine Eon, Jessica Breadsell, Gregory Morrison, Joshua Byrne

\section{Introduction}

Energy reduction in residential buildings is considered to be one of the most straight forward ways of reducing carbon emissions. Many OECD countries and jurisdictions currently have energy efficiency policies in place, usually targeting the improvement of building shells for higher thermal efficiency, the installation of energy efficient appliances and the adoption of renewable energy. While valuable, these measures do not provide a holistic approach to the energy reduction challenge.

Previous research has shown that highly energy efficient or net zero energy buildings (i.e. buildings that produce as much energy through onsite renewable energy generation as they consume) do not achieve their full potential despite performing better than conventional buildings (Watson, 2015). There are a number of reasons for this underperformance, including poor construction practices; nevertheless, the unpredictability of occupancy constitutes an important factor. Previous research has shown that identical buildings can consume very different levels of resources mostly due to their occupants, which may not only own different appliances, but are also likely to follow different routines and have distinct behaviour patterns (Hansen, 2016; Strengers and Nicholls, 2017). Household and occupants level of skills and understanding of specific technologies also impact the dwelling's metabolic system - i.e. its resource flows (Pettersen et al., 2017).

A popular approach to decreasing the negative impacts of occupancy on residential energy use has been through the implementation of methods grounded in social psychology. These 
methods attempt to persuade change without consideration of the full picture; that is, people's environments, lifestyles, needs and everyday practices. An alternative and more contemporary approach to influencing residents is based on social practice theory (Schatzki, 2002; Warde, 2005; Shove, 2007; Røpke, 2009). Rather than targeting the individual in isolation, practice theory emphasises people's daily activity patterns and interactions with their technology, dwelling and urban infrastructure contexts. Practice theory also considers skills and abilities and intrinsic motivations for undertaking certain practices (Eon et al., 2018a). As an integral part of the home, occupants need to be considered when innovative buildings and associated technologies are planned for, designed and constructed. Energy reductions are more successful when enabled through the integration of good design into the home system (Smale, van Vliet and Spaargaren, 2017).

This chapter introduces the concept of home system of practice (HSOP) and discusses how this notion can be useful in shifting energy consumption in residential buildings in both the long and the short term. Firstly, the layers that form the home system are briefly described, then the more customary theories for changing consumer behaviour are reviewed and finally we advance how effective comprehension of the HSOP can act as an enabler for achieving more sustainable built environments - as designed as well as operated.

\section{The home system}

The home is a complex environment which can be conceived as a combination of three systems: the physical, the metabolic and the social systems (Figure 1). The physical system is an ensemble of objects, technologies and infrastructure that make up the building. The social system consists of the building occupants, who are affected by their cultural beliefs, values, knowledge, skills, their personal networks and wider society. The metabolic system involves the movements of materials, water and energy, which flow through pipes and cables and are 
internally processed through the daily operation of appliances and fittings. The metabolic system is affected by its interactions with the physical and the social systems (Eon et al. 2018a). To effectively reduce energy use in the home, it is necessary to influence both the physical and the social systems.

< Figure 1. The home system complex: social, metabolic, and physical systems (adapted from Eon et al., 2018a).>

Effective technologies for reducing energy consumption and carbon emissions in the residential sector are all well-studied and increasingly affordable. These include energy efficient building envelope design and low carbon materials, energy efficient appliances and renewable energy systems. The social system, on the other hand, is not so well understood and is often ignored. It is typically not until after new building technologies have been implemented and failed in their objectives to reduce energy consumption that the occupants come into focus and an attempt is made toward shifting their customary behaviours (Gram-Hanssen et al., 2017).

\section{Traditional methods for shifting behaviour}

Conventional methods for shifting occupant behaviour are grounded in four major psychosocial theories: cognitive dissonance, planned behaviour, normative conduct and habitual behaviour. The theory of cognitive dissonance posits that individuals are conflicted when they recognize that their values and their actions are inconsistent and work to realign them, resulting in either a change in behaviour or a change in attitude (Festinger, 1957). The theory of planned behaviour proposes that behaviours are a product of attitudes, social norms and the perceived control individuals have over the outcomes of their own actions (Ajzen, 1991). A change in behaviour would therefore require an alteration of these three factors. The theory of normative 
conduct suggests that individuals are influenced by wider societal norms and unspoken judgements (Cialdini et al., 1991, Schultz et al., 2007). Information about customary community behaviours and expectations is believed to shift individual behaviours. Finally, the theory of habitual behaviour considers that behaviours become automatic and unconscious when repeated regularly (Aarts et al., 1998). Hence, breaking established habits would require either a drastic change in context or frequent prompts (Steg and Vlek, 2009).

Common interventions are based on the above theories and include: the provision of information and feedback to increase awareness; the delivery of social norms to make accepted and unaccepted behaviours explicit; the request for a clear commitment or highlighting of hidden personal values to promote cognitive dissonance; and the delivery of prompts to break established undesirable habits (Abrahamse et al., 2005, McKenzie-Mohr, 2011).

These interventions have been deployed in research and practice through a range of approaches that generally fall into three categories classified here as social, technological and knowledgebased interventions (Table 1). The most successful interventions often integrate social, technical and knowledge-based methods together, such as through real-time feedback, coaching and information campaigns; however, there are few long-term studies of the kind.

The use of in-home displays or dashboards, have become increasingly popular for conveying information and prompts and seeking some response from the users. A driver here is that house metering technology has become more accessible and enables real-time feedback that is often not possible through more conventional methods such as coaching or personalised letters. Nonetheless, opinions about the effectiveness of feedback displays is divided; some researchers claim that they are effective in the reduction of resource use and identification of faulty equipment (Berry et al., 2017, Stromback et al., 2011), while others have found that they are not effective in the long term as they do not become embedded into user's routines and their 
use is discontinued after the novelty wears off (Brynjarsdottir et al., 2012, Hargreaves et al., 2013). It is also argued that feedback systems are developed by technologists and do not necessarily meet user requirements.

< Table 1. Conventional methods for shifting behaviour.>

Information campaigns through media advertisements are popular amongst governing bodies and utilities (e.g. water and electricity providers), who see this as a means for attempting to rapidly 'broadcast' awareness through society.

Social psycho-social theories address resource use from a top-down perspective, persuading home occupants to change individual attitudes, perceptions and behaviours based on information being provided through the methods on Table 1. Nevertheless, behaviours are influenced by the wider society and culture (Stephenson et al., 2010, Shove et al., 2015) and changing them entails a societal transition, being based on a more systemic approach.

\section{The home system of practice}

The concept of HSOP (Eon et al., 2018a) emerged from practice theory, which offers an alternative to understanding and shifting actions by focusing on everyday practices as opposed to resident behaviour, knowledge and attitudes (Shove et al., 2007, Schatzki, 1996). Practice theory suggests that individuals do not use energy resources directly, but rather as instruments to achieve specific outcomes (Hargreaves, 2011). For instance, energy is used in the practice of cooking with the objective of preparing food for consumption; water is used in the practice of personal hygiene through a shower; and driving a car is used in multiple practices such as shopping, getting to work and dropping children at school.

Practices conducted by users are affected by three elements: meaning, skill and technology (Schatzki, 1996, Gram-Hanssen, 2014). Meaning is the reason behind the execution of a practice; skill is the understanding of how to execute the practice; and technology encompasses 
the objects and infrastructure necessary to undertake the practice. It follows that affecting one or more of these elements should result in a modification of the practice and subsequently the resource use, enabling (as opposed to persuading) occupants to save energy while continuing to meet their needs (Brynjarsdottir et al., 2012).

As technologies and infrastructures evolve and are adopted, existing social practices become obsolete and are replaced by new ones (Shove et al., 2015). Practices are also place and time dependent, being adapted to the configuration of different settings and circumstances.

The repetition of practices in a habitual routine become interdependent and interlocked (i.e. interconnected) in a system of practice (SOP; Watson, 2012). For instance, the practice of composting is interlocked with the practice of food preparation; in other words, composting cannot exist unless food waste is generated. Likewise, practices are often reproduced in a sequential manner, interlocking with preceding and subsequent practices (Eon et al., 2018a). For instance, the practices of showering, eating breakfast and driving to work are all constrained by the practice of working and its schedules (Southerton 2013; Torriti 2017).

In a home occupied by multiple individuals, each individual possesses a unique SOP. These SOPs interlock with each other as some practices are shared between individuals (e.g. eating a meal), occur sequentially (e.g. showering) or take place as a consequence of another set of household activities (e.g. cleaning up after children). This network of SOPs in the home forms a HSOP (Figure 2), which is part of the social system of the home. The HSOP can be regarded as a form of home equilibrium, and while complex, works in harmony to achieve desired outcomes for household members (Eon, 2017).

<Figure 2. Interlocked practices and routines in the HSOP (adapted from Eon, 2017).> 


\section{Shifting energy consumption within the HSOP}

Shifting domestic energy consumption requires a deep understanding of the HSOP; that is, the interconnections that exist between an individuals' own practices as well as within the HSOP as a whole and incorporating the meanings, skills and technologies-in-use behind those practices that use energy. These are explored in the following section.

\subsection{Implications of the interlocking of practices}

Practices can have different degrees of interlocking; being highly or lightly interlocked in the HSOP. Research has shown that practices occurring during workdays usually happen within tight timeframes as they are limited by predetermined activities, such as work or school and their timetables (Torriti 2017; Eon et al. 2018a). In such cases, practices have a high degree of interlocking and are more strongly bound in the home equilibrium. This means that altering these practices, their times, duration or order, can prove hard as not only the practices themselves need to be affected, but all other interlocked practices in the HSOP. Conversely, practices that occur during non-working days or that are not bound to recurring scheduled activities, are deemed to be lightly interlocked. These are usually more flexible, have varying timetables and durations and are less dependent on the household routine, being therefore potentially easier to modify.

Whenever a new practice is introduced in the home, interlocked practices need to be re-aligned so that the new practice becomes incorporated in the HSOP (Figure 3). Unless a new home equilibrium is reached and the new practice becomes embedded in the HSOP, it is not adopted by the home occupant. This tends to be the case with persuasive approaches, such as the use of feedback systems. While occupants usually value information about their energy use, the practice of accessing the feedback system platform fails to become integrated in the HSOP and 
does not have significant lasting effects. Reasons can vary from lack of time and being busy, to forgetting about it (Eon et al., 2018b).

$<$ Figure 3. Conceptual diagram of the equilibrium of practices in the home. (a) Original home equilibrium; (b) Destabilisation of the home equilibrium through the introduction of a new practice; (c) Realignment of practices and establishment of a new home equilibrium (adapted from Eon, 2017).>

A HSOP realignment does not necessarily mean that practices themselves are affected. While the timing of practices may realign and reach a new equilibrium, the meanings, skills and technologies of the practices may remain the same; that is, the manner in which occupants perform specific practices to achieve a particular meaning may not be affected. As resources are consumed indirectly through the performance of practices, the realignment of the elements may result in the same level of resources being consumed during the practice (Eon et al., 2018a).

\subsection{The implication of the meaning of practices}

The technology and related skills required for carrying out a certain practice are relatively constant over time in a specific context. In contrast, more than one meaning can be attributed to the same practice (Shove, 2003). For example, research has shown that the practice of using a heater can be associated with multiple meanings or associations; e.g. warmth, comfort, health and habit (Eon et al., 2018c). Different meanings impact differently on the resources required for the execution of a particular practice. For showering, it has been shown that meanings such as cleanliness or refreshment are associated with shorter showers and thus less energy and 
water consumption. Meanings such as warmth and relaxation, on the other hand, are associated with longer showers and therefore higher resource use (Breadsell et al. forthcoming).

Individuals who assign multiple meanings to the same practice and thus use varying amounts of resources at each instance may be able to make a conscious decision to only adopt one of its more resource-efficient meanings and associated consumption patterns. Conversely, individuals who have one sole meaning for a practice may not be willing or may not have the skills to reduce the associated resource use.

Requesting that occupants change the meanings of their practices without providing a suitable alternative to meet the same need is challenging. If the heater is turned on with the purpose of comfort, it may be hard for its use to be reduced without negatively impacting the user's lifestyle. Information campaigns that have urged consumers to reduce their shower lengths, for example, have failed as they have not properly addressed the meaning behind the practice. In this case, the adoption of an alternative technology, such as a more efficient shower head or water heating appliance, may be a more suitable solution for the purpose of reducing energy consumption in the home.

\subsection{Enabling change in the HSOP}

To enable change to consumption patterns in the home, one of three scenarios needs to take place:

- a new practice needs to be incorporated in the HSOP leading to a new home equilibrium;

- $\quad$ one of the elements of the targeted practice needs to be modified; or

- a practice needs to be dis-interlocked or disconnected from the HSOP in order to act independently of the other occupants. 
Individuals that perform habitual and highly interlocked practices are unlikely to change them unless there is a major modification in context (e.g. a change in lifestyle, family structure or to the technology element of the practice) causing practices to realign. Practice theorists posit that rather than persuading individuals to change behaviour and realign existing routines, the elements of practice should be targeted (Spurling et al. 2013; Eon et al. 2018a).

A change in meaning can be challenging as it is the reason behind the execution of a practice (Shove et al., 2012); that is, meaning relates to a need that an individual wants to fulfil and that directly impacts on the perceptions of lifestyle, comfort and wellbeing. The skill associated with a practice is learned through the observation of other practitioners over the years, being family, society and culture dependent (Gram-Hanssen, 2010, Scott et al., 2012). Affecting skills might therefore entail a shift in an individual's perception; which is also problematic to achieve in the short term. In contrast, the technology element of the practice can be more easily adjusted as it usually consists of a one-off change that does not affect the HSOP nor has a major impact on established habits and comfort (Eon et al., 2018b). This is supported by research that suggests that consumers are favourable to more efficient technologies but perceive convenience, practicality and cost as factors in take-up (Dolnicar and Hurlimann, 2010). Technology changes can often be made when an individual is moving to a new house or purchasing new appliances.

Innovative technologies must be designed to meet occupant needs and be properly understood to avoid the risk of generating undesired rebound effects (Wolff et al., 2017). Scott et al. (2012) propose that enabling change in practice should be conducted through practice-oriented design, comprising the following steps: understanding the baseline practices; challenging the status quo by identifying alternative solutions; and co-creating solutions with the users. This process encourages the development of innovative technologies capable of meeting users' needs 
including more efficient use of resources. An example of this is the redesigning of bathing practices by Kuijer, Jong (2009).

Another solution to enable energy reduction in the home is through unlocking practices from the HSOP; that is, making them independent of other occupants or other systems of (low carbon) energy supply. This can be achieved through the use of automation that can be built into to the physical systems of the home. For instance, the practices of dishwashing, clothes washing and pool cleaning can be automated to occur at times when renewable energy is being generated but when occupants are not necessarily present to carry out the task themselves. Battery storage will expand the opportunity here. Similarly, appliances on standby can be programmed to be switched off when not in use and air conditioners can be controlled to function optimally in line with external factors such as temperature. While the aforementioned practices can be executed manually, they are considered a hassle by occupants and seldom integrated into established routines (Hobman, Stenner and Frederiks, 2017).

Manual practices are bound in space and time in a tightly interlocked routine and can be changed through a change in the elements. Automated practices, in contrast, are bound only in space as they can function at flexible times and operate in conformity with the physical home system and independently from the HSOP. For instance, a timer could be installed to the reticulation system so it is independent of the rest of the HSOP. Careful consideration needs to be given when designing and deploying automated technologies as they are required to meet occupant needs and skills to work effectively and produce the desired outcomes for the household - and society more broadly.

\section{Conclusion}

This chapter outlined the theoretical concept of the HSOP, which can be used to provide a deeper understanding of the social system associated with the home and inform solutions for 
enabling energy reduction at a household level. More traditional methods have attempted to persuade occupants to change through the use of information campaigns and feedback technology. However, their effects are usually short-lived. Interventions aimed at affecting specific practices may ignore the underlying reasons for these practices and their interconnectedness within the home system. The effective modification of occupant behaviours and everyday practices requires a holistic understanding of the HSOP, which includes occupant practices, routines, and their interconnections.

Whilst HSOPs can be realigned, they constitute a challenging task without a more fundamental change in context. Affecting the technology elements of practices on the other hand may be more readily accepted as they do not impact occupant meaning, comfort and lifestyle. This extends to the use of automated technology that can be operated independently of users. Even though the idea of utilising automation for improved house performance is not new, aligning it with the concept of HSOP can assist with improved design, deployment and adoption of technologies that enable low carbon practices.

\section{Acknowledgement}

This research was funded by the CRC for Low Carbon Living Ltd supported by the Cooperative Research Centers program, an Australian Government initiative.

\section{References}

Aarts, H., Verplanken, B. \& Van Knippenberg, A. 1998. Predicting Behavior From Actions in the Past: Repeated Decision Making or a Matter of Habit? Journal of Applied Social Psychology, 28, 1355-1374.

Abrahamse, W., Steg, L., Vlek, C. \& Rothengatter, T. 2005. A review of intervention studies aimed at household energy conservation. Journal of Environmental Psychology, 25, 273-291. 
Ajzen, I. 1991. The theory of planned behavior. Organizational Behavior and Human Decision Processes, 50, 179-211.

Berry, S., Whaley, D., Saman, W. \& Davidson, K. 2017. Finding faults and influencing consumption: the role of in-home energy feedback displays in managing high-tech homes. Energy Efficiency, 10, 787-807.

Breadsell, J. K., Eon, C., Morrison, G. M. \& Kashima, Y. forthcoming. A Home System of Practice Analaysis of Low Carbon Precinct Residents, Environment and Planning B: Urban Analytics and City Science.

Brynjarsdottir, H., H, M., Kansson, Pierce, J., Baumer, E., Disalvo, C. \& Sengers, P. 2012. Sustainably unpersuaded: how persuasion narrows our vision of sustainability. Proceedings of the SIGCHI Conference on Human Factors in Computing Systems. Austin, Texas, USA: ACM.

Cialdini, R. B., Kallgren, C. A. \& Reno, R. R. 1991. A Focus Theory of Normative Conduct: A Theoretical Refinement and Reevaluation of the Role of Norms in Human Behavior. In: Mark, P. Z. (ed.) Advances in Experimental Social Psychology. Academic Press.

Dolnicar, S. \& Hurlimann, A. 2010. Australians' Water Conservation Behaviours and Attitudes. Australasian Journal of Water Resources, 14, 43-53.

Eon, C. 2017. The home system of practice. PhD, Curtin University.

Eon, C., Breadsell, J. K., Morrison, G. M. \& Byrne, J. 2018a. The home as a system of practice and its implications for energy and water metabolism. Sustainable Production and Consumption, 13, 48-59.

Eon, C., Liu, X., Morrison, G. M. \& Byrne, J. 2018b. Influencing energy and water use within a home system of practice. Energy and Buildings, 158, 848-860. 
Eon, C., Morrison, G. M. \& Byrne, J. 2018c. The influence of design and everyday practices on individual heating and cooling behaviour in residential homes. Energy Efficiency, 11, 273293.

Festinger, L. 1957. A theory of cognitive dissonance, United States of America, Row, Peterson and Company.

Gram-Hanssen, K. 2010. Residential heat comfort practices: understanding users. Building Research \& Information, 38, 175-186.

Gram-Hanssen, K. 2014. New needs for better understanding of household's energy consumption - behaviour, lifestyle or practices? Architectural Engineering and Design Management, 10, 91-107.

Gram-Hanssen, K., Heidenstrom, N., Vitterso, G., Madsen, L. V. \& Jacobsen, M. H. 2017. Selling and installing heat pumps: influencing household practices, Building Research \& Information, 45(4), 359-370.

Hansen, A. R. 2016.The social structure of heat consumption in Denmark: New interpretations from quantitative analysis, Energy Research and Social Science, 11, 109-118.

Hargreaves, T. 2011. Practice-ing behaviour change: Applying social practice theory to proenvironmental behaviour change. Journal of Consumer Culture, 11, 79-99.

Hargreaves, T., Nye, M. \& Burgess, J. 2013. Keeping energy visible? Exploring how householders interact with feedback from smart energy monitors in the longer term. Energy Policy, 52, 126-134.

Hobman, E. V., Stenner, K. \& Frederiks, E. R. 2017. Exploring everyday energy usage practices in Australian households: A qualitative analysis. Energies, 10(1332). 
Kuijer, L.; Jong, A.M. De. 2009. Design as an instrument to bring about behavioral change. In Proceedings of the European Council for an Energy Efficient Economy- Energy Efficiency and Behaviour Conference.

Mckenzie-Mohr, D. 2011. Fostering sustainable behavior: an introduction to community-based social marketing / Doug McKenzie-Mohr, New York, New York: New Society Publishers.

Pettersen, I. N., Verhulst, E., Valle Kinloch, R., Junghans, A. \& Berker, T. 2017.Ambitions at work: Professional practices and the energy performance of non-residential buildings in Norway.Energy Research \& Social Science. 32,112-120.

Ropke, I. 2009.Theories of practice — New inspiration for ecological economic studies on consumption. Ecological Economics. 68(10).2490-2497.

Schatzki, T. R. 1996. Social Practices: A Wittgensteinian Approach to Human Activity and the Social, New York, Cambridge University Press.

Schatzki, T. 2002. The Site of the Social. Pennsylvania: The Pennsylvania State University Press.

Schultz, Nolan, Cialdini, Goldstein \& Griskevicius 2007. The Constructive, Destructive, and Reconstructive Power of Social Norms. Psychological Science (Wiley-Blackwell), 18, 429434.

Scott, K., Bakker, C. \& Quist, J. 2012. Designing change by living change. Design Studies, 33, 279-297.

Shove, E. 2003. Converging Conventions of Comfort, Cleanliness and Convenience. Journal of Consumer Policy, 26, 395-418.

Shove, E., Watson, M., Hand, M., Ingram, J., 2007. The Design of Everyday Life. Berg, Oxford \& New York 
Shove, E., Pantzar, M. \& Watson, M. 2012. The dynamics of social practice: everyday life and how it changes, London, SAGE Publications Ltd.

Shove, E., Watson, M., Hand, M. \& Ingram, J. 2007. The design of everyday life, Oxford and New York, Berg.

Shove, E., Watson, M. \& Spurling, N. 2015. Conceptualizing connections: Energy demand, infrastructures and social practices. European Journal of Social Theory, 18, 274-287.

Smale, R., Van Vliet, B. \& Spaargaren, G. 2017. When social practices meet smart grids: Flexibility, grid management, and domestic consumption in The Netherlands.Energy Research and Social Science. 34132-140.

Southerton, D. 2013. Habits, routines and temporalities of consumption: From individual behaviours to the reproduction of everyday practices.Time \& Society, 22(3),335-355.

Sorrell, S., Dimitropoulos, J. \& Sommerville, M. 2009. Empirical estimates of the direct rebound effect: A review. Energy Policy, 37, 1356-1371.

Spurling, N., Mcmeekin, A., Shove, E., Southerton, D. \& Welch, D. 2013.Interventions in practice: re-framing policy approaches to consumer behaviour. Sustainable Practices Research Group < http://www.sprg.ac.uk/uploads/sprg-report-sept-2013.pdf>

Steg, L. \& Vlek, C. 2009. Encouraging pro-environmental behaviour: An integrative review and research agenda. Journal of Environmental Psychology, 29, 309-317.

Stephenson, J., Barton, B., Carrington, G., Gnoth, D., Lawson, R. \& Thorsnes, P. 2010. Energy cultures: A framework for understanding energy behaviours. Energy Policy, 38, 6120-6129.

Strengers, Y. \& Nicholls, L. 2017. Convenience and energy consumption in the smart home of the future: Industry visions from Australia and beyond. Energy Research \& Social Science. 32, 86-93. 
Stromback, J., Dromacque, C., Yassin, M. H. \& Vaasaett, G. E. T. T. 2011. The potential of smart meter enabled programs to increase energy and systems efficiency: a mass pilot comparison Short name: Empower Demand. Vaasa ETT.

Torriti, J. 2017. Understanding the timing of energy demand through time use data: Time of the day dependence of social practices and energy demand. Energy Research \& Social Science. 25,37-47.

Warde, A. 2005. Consumption and Theories of Practice. Journal of Consumer Culture, 5(2), $131-153$.

Watson, M. 2012. How theories of practice can inform transition to a decarbonised transport system. Journal of Transport Geography, 24, 488-496.

Watson, K. J. 2015. Understanding the role of building management in the low-energy performance of passive sustainable design: Practices of natural ventilation in a UK office building. Indoor and Built Environment, 24(7),999-1009.

Wolff, A., Weber, I., Gill, B., Schubert, J. \& Schneider, M. 2017. Tackling the interplay of occupants' heating practices and building physics: Insights from a German mixed methods study. Energy Research and Social Science, 32,65-75. 